\title{
-Review- \\ Germ Cell Differentiation in Culture
}

\author{
Toshiaki Noce ${ }^{1}$ \\ ${ }^{1}$ Laboratory of Reproductive Biology, Mitsubishi Kasei Institute of Life Sciences, 11 Minami-ooya, \\ Machida-shi, 194-8511 Tokyo, Japan
}

The generation of animals cloned by means of the nuclear transfer technique indicates that somatic cell nuclei can be reprogrammed when they are transplanted into the egg cytoplasm-in other words, the transplanted somatic nuclei can be induced to return to a pluripotent state similar to that of the nuclei of fertilized eggs. Therefore, the potential for both reprogramming and pluripotency is retained in germ cells throughout their development. For example, embryonic germ (EG) cells that are generated from primordial germ cells (PGCs) show similar pluripotency to the undifferentiated embryonic stem (ES) cells that originate in the inner cell mass of blastocyst embryos in mice [1]. And some teratomas that generate differentiated cells of various types-from all three primary germ layers-originate in PGCs in the embryonic gonads [2]. Therefore, germ cells must have the ability to be reprogrammed, as well as to differentiate to form gametes. So how do germ cells acquire these characteristics, and how are they maintained during development? The discovery of the molecular and cellular mechanisms that are involved in these processes will provide important insights not only for basic research into reproductive biology but also for the development of new techniques for regenerative medical treatments. There is an urgent need to develop in vitro systems that can model the entire developmental process-from pluripotent stem cells to functional sperm and eggs-to further our understanding of the mechanisms of germ-cell differentiation.

\section{In Vitro Differentiation of Primordial Germ Cells}

In mammals-unlike other animal species, such as frogs and flies-the germ cells are not predetermined by

Received: March 31, 2003

Accepted: May 18, 2003

e-mail: noce@libra.ls.m-kagaku.co.jp inherited maternal factors. Germ-cell determination in mice occurs during the mid-gastrulation stage, approximately 6.5 days after implantation. Previous studies have clearly shown that PGC determination depends on the inductive effects of trophoblast tissue in embryos at the egg-cylinder stage, and that only a limited number of cells in the proximal region of the epiblast differentiate to become PGCs [3, 4]. Germ-cell differentiation from ES cells in culture has previously been used to investigate the mechanisms by which germ cells segregate from the pluripotent epiblast cells. This was regarded as the most suitable model system, because of the high reproducibility of ES cells in culture, and the unlimited number of cells that can be produced. It is well established that ES cells can differentiate into all cell lineages when they are introduced into host blastocysts, and they can also generate various somatic cell lineages in vitro. Therefore, as ES cells can contribute to the germline of a host embryo, in theory they should also be able to produce germ cells in vitro. Nevertheless, a crucial difficulty in such culture systems is distinguishing between undifferentiated ES cells and differentiating PGCs, because they share many common characteristics, including the expression of Oct $3 / 4$, SSEA 1 antigen and strong alkaline phosphatase (APase) staining $[5,6]$. One gene that is expressed in differentiating germ cells-mouse vasa homolog $(M v h)$ - provides a specific marker to distinguish the cells of the germ-cell lineage from both undifferentiated cells and somatic cells. The vasa gene was originally identified in Drosophila as a member of a group of maternal factors that are required for the formation of germplasm and germ-precursor pole cells [7]. The vasa gene encodes DEAD-family ATPdependent RNA helicase. In mice, the expression of $\mathrm{MVH}$ protein is not detectable in embryos before 9.5 days. MVH-positive cells are first detected in PGCs just after they colonize the embryonic gonads, in both males and females. Subsequently, MVH expression persists in germ cells that are undergoing gametogenesis until 
they reach the post-meiotic stages-spermatids in males and primary oocytes in females [8]. This specific expression pattern is also seen in the vasa homolog gene in humans [9].

The most suitable in vitro method for the detection of PGC formation is the visualization of PGC-specific gene expression by establishing knock-in ES cell lines. MVH expression can be monitored with an ES cell line in which the $M v h$ gene is replaced by a lacZ gene through homologous recombination-a color change induced by exogenous $\beta$-galactosidase activity can identify MVHpositive PGCs. By this method we demonstrated the presence of MVH-positive PGCs during the formation of embryoid bodies that were generated from Mvh-lacZ knock-in ES cells (Fig. 1). This indicates that ES cells can differentiate to form germ-cell lineages, as well as somatic cell lineages, in culture. This technique will provide insights into several issues concerning the nature of germ-cell development. An additional advantage of this in vitro system is that the exact number of induced PGCs can be quantified by cellsorting analysis.

As described earlier, germ-cell determination in mice occurs at the onset of gastrulation, and germ-cell formation is dependent on the inductive influence of extra-embryonic ectodermal cells-the trophoblast precursor cells. BMP4, a member of the TGF- $\beta$ family of morphogenic factors that is produced by trophoblast precursors, is a good candidate for an inducing factor, because PGCs are not formed in knockout mice for the Bmp4 gene [10]. In addition, recent work has shown an inductive function of BMP4 in vitro-quantitative analysis with a cell-sorter machine has indicated that co-culturing ES cells with trophoblast or BMP4producing cells greatly enhances the generation of germ cells from ES cells in culture (Toyooka et al., in preparation). An interesting aspect of this inductive effect is that the formation of tight cell aggregates, consisting of both ES cells and effecter cells, is crucial for induction-no induction occurs if ES cells are cocultured on a feeder layer of effecter cells alone. Therefore, it is likely that germ-cell differentiation is dependent not only on inducing factors such as BMP4, but also on the microenvironment that is provided by tight cell aggregates, which probably exist within the epiblast cell mass in vivo. Another interesting feature is the proportion of the PGCs in the cell mass. Although proximal epiblast cells acquire the capacity for PGCs through stimulation by BMP4, the origins of PGC founder cells seem to be randomly distributed in the proximal region of the epiblast [3]. Only a small number

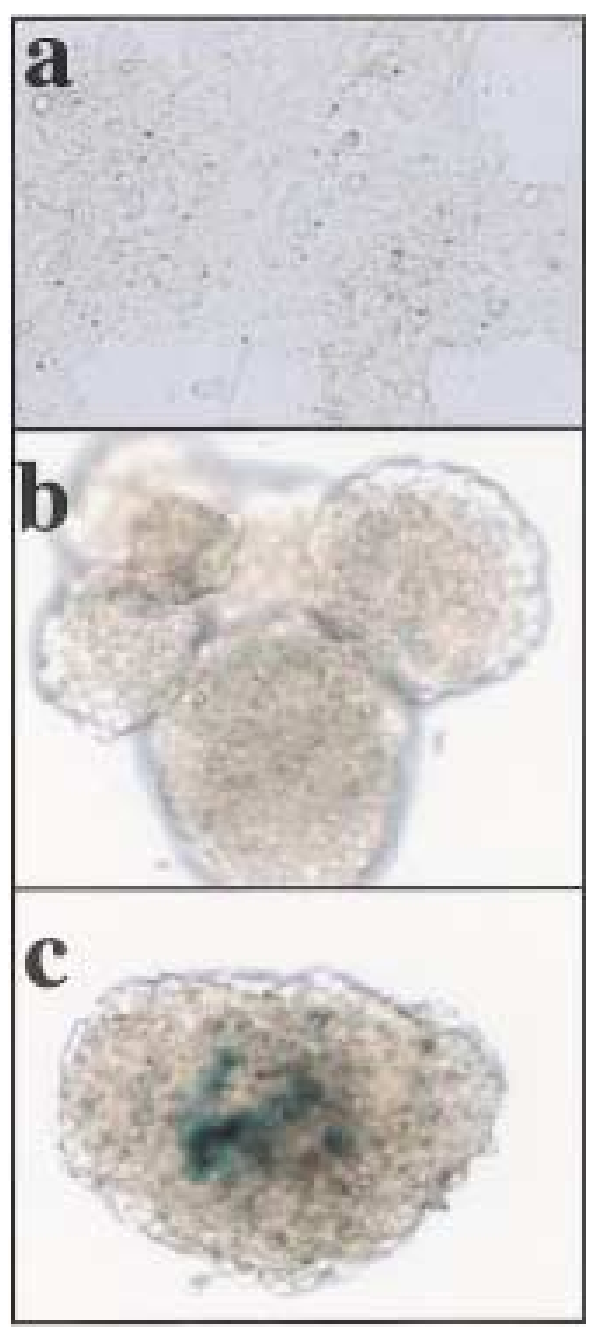

Fig. 1. In vitro differentiation by means of embryoid body formation of knockin ES cells. Staining images by $\beta$ galactosidase activity of Mvh-lacZ knock-in ES cells; undifferentiated state (a), 2 days (b) and 5 days after embryoid body formation (c). LacZ positive (Mvh-positive) PGCs appeared after 3-5 days.

of the epiblast cells, approximately 45 cells per embryo, become PGCs. This is also the case in vitro. Even in a homogenous culture, such as a uniform mixture of ES cells and effecter cells, only a limited population of the ES cells become germ cells (1-3\% of the ES cells). This indicates that ES cells, as well as epiblast cells, might have some variation in their tendency to become germline cells. Future studies with in vitro culture systems will provide insights into this issue.

ES $(X Y)$ cells that are derived from PGCs in culture 


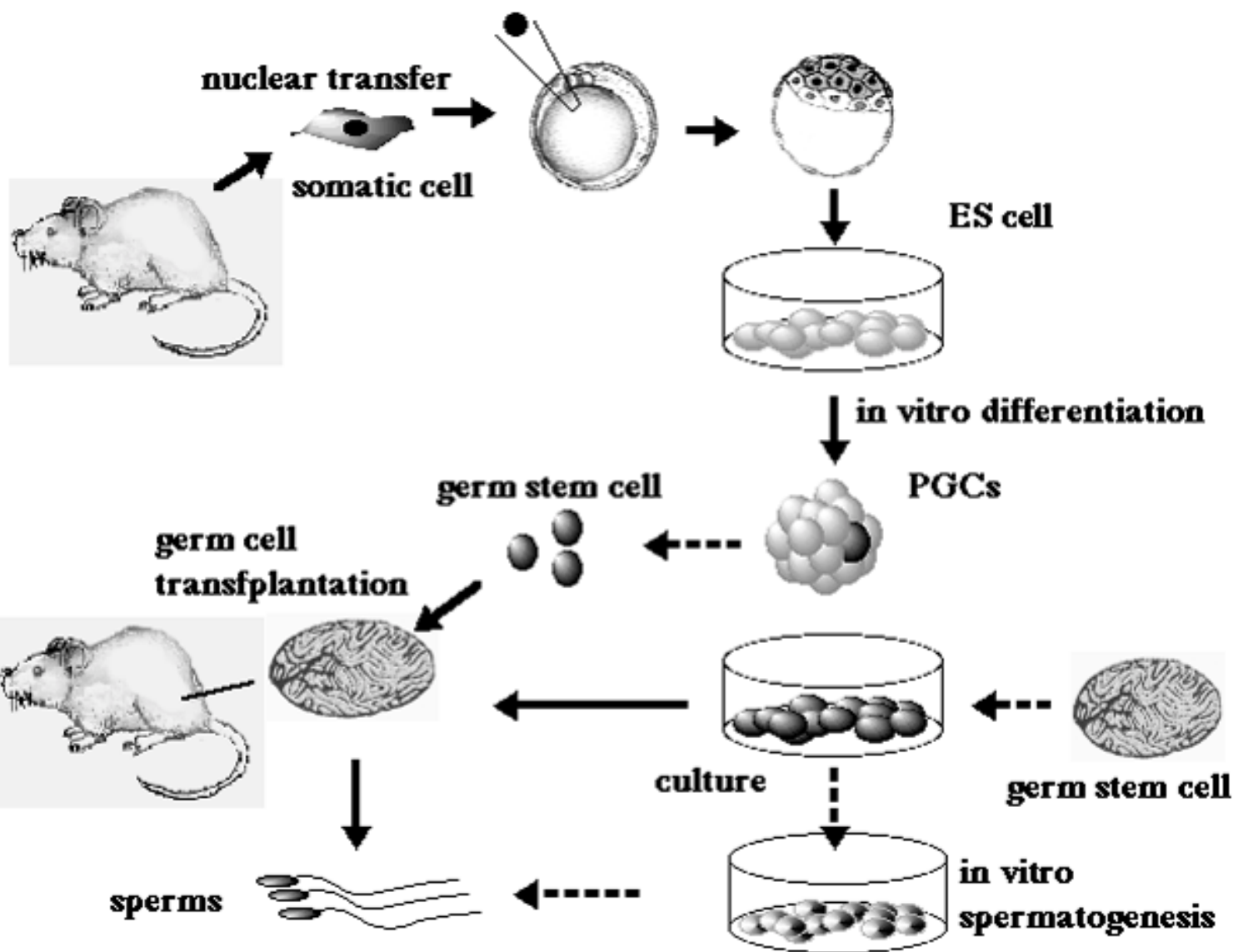

Fig. 2. Predictable approaches for generating functional gametes by using a culture system.

can undergo spermatogenesis if they are transplanted into host adult testes after having formed a coaggregate with gonadal cells prepared from male embryonic gonads (Toyooka et al. in preparation); this indicates that the ES-derived germ cells are at least equivalent to the PGCs that colonize embryonic gonads. In theory, ES-derived germ cells will also be able to produce oocytes if they are transplanted into ovaries after having co-aggregated with female gonadal cells. Nevertheless, the results of these transplantation experiments do not imply that ES cells can differentiate autonomously to form sperm and eggs in culturegonadal supporting cells are always necessary for the production of gametes from ES cells. In order to improve this model system, it will be crucial to reproduce the developmental conditions in the embryonic gonads that induce PGCs to form spermatogonial stem cells or primary oocytes. If germstem cells can be established in culture from ES cells by means of a testes cell transplantation technique, as described in the next section, it will become much easier to generate ES cell-derived functional sperm and to use these to produce offspring through natural mating.

\section{Approaches for Making Functional Sperm and Eggs by Means of an In Vitro Culture System}

Several recent efforts to culture and transplant germ cells have revealed new approaches to the generation of functional gametes in vitro (Fig. 2). The testes cell transplantation method established by Brinster and colleagues has defined several characteristics of spermatogonial stem cells $[11,12]$. Spermatogonial stem cells in mammals are found on the basement membrane of the seminiferous tubule, at an average frequency of 1 per 5,000 testes cells. These cells selfrenew throughout their lifetime, and continuously produce unipotent daughter cells that differentiate into sperm. Brinster and colleagues showed that if stem cells from the testes of a donor were transplanted into the seminiferous tubules of an infertile recipient mouse, 
the donor cells colonized the host testes and spermatogenesis was resumed. This colonization of donor-derived stem cells was also found when cells from late embryonic gonads (E18) and immature testes were transplanted. Furthermore, these authors showed that spermatogonial stem cells could survive in culture for more than 3 months without the loss of function. These findings indicate that if spermatogonial stem cells can be proliferated in culture, rather than in an ES cell system, experimental genetic modifications of the mouse germline-including targeted gene disruptionwill be achieved more easily and rapidly. This technique might be applicable to many animal species, and could make it possible to clinically treat male infertility in humans.

Second, a recent study by Feng et al. [13] established spermatogonial cell lines by telomerase immortalization, and found that the immortalized cells could differentiate into spermatids in vitro in the absence of supporting cells (such as Sertoli cells). In this study, differentiation was induced by the addition of stem-cell factor (SCF). One week after induction, approximately $40 \%$ of the cells had developed into spermatocytes. After 3 weeks, more than $50 \%$ of the cells had differentiated into spermatids, but no spermatozoa were observed in this culture. By contrast, in a previous study of in vitro spermatogenesis that used immortalized Sertoli cells as a feeder layer, the testicular germ cells differentiated into mature spermatozoa [14]. In this experiment, the testes cells that were prepared from wild-type mice only survived for a maximum of 2 weeks in vitro; therefore it is likely that the spermatazoa were generated from cells that had already differentiated into spermatids before they were cultured, but these results are still of interest as they show that spermiogenesis can take place in vitro.

Almost all of the previous efforts to establish a culture system that models the entire process of spermatogenesis in mammals-from stem cells to spermatozoa-have failed, even when organ or tissue culture has been used. It is clear that something is missing from the culture media that is essential for the long-term survival and differentiation of spermatogenic cells. By contrast, a recent report showed that complete spermatogenesis occurred when tissues from the testes of immature mice, pigs and other species were grafted under the skin of immunodeficient host mice [15]. As a consequence of the differences between the results of in vitro cultures and in vivo grafts, it seems likely that normal spermatogenesis requires the combined effects of gonadal hormones. The feedback control of endocrine factors in the hypothalamus, pituitary and testes plays a crucial role in the process of spermatogenesis in vivo, which obviously does not occur in culture. Therefore, it is possible that further improvement of the culture techniques will enable the complete process of spermatogenesis to be reproduced in vitro.

Germ cells are specialized to transmit all of the genomic information of an organism to the next generation; in other words, the genetic information written in the genome DNA functions normally only when it is transmitted through the development of germline cells. In order to fully understand the mysterious properties of germline cells, further efforts to improve in vitro culture systems will be required.

\section{Conclusion}

Germ cells are the only cells that transmit genetic information and developmental pluripotency from one generation to the next. Cloned animals can be generated by the transplantation of somatic nuclei into unfertilized eggs, which shows that cytoplasmic factors in the oocytes are able to induce pluripotency in somatic nuclei. Therefore, reprogramming activity seems to be maintained throughout the development of germline cells. Numerous studies have attempted to investigate the ways in which germ cells are specialized for reproduction-mainly by searching for genes with germline-specific expression. But the lack of culture systems that can successfully model cell differentiation in vitro has hampered efforts to understand the molecular basis of germ-cell specificity and differentiation. Several recent studies involving the culture and transplantation of germ cells have highlighted potential methods to solve these problems.

\section{References}

1) Labosky, P.A., Barlow, D.P. and Hogan, B.L. (1994): Mouse embryonic germ cell lines: transmission through the germline and differences in the methylation imprint of insulin-like growth factor 2 receptor (Igf2r) gene compared with embryonic stem cell lines. Development, 120, 31973204.

2) Stevens, C.L. (1978): Totipotent cells of parthenogenic origin in a chimeric mouse. Nature, 276, 266-267.

3) Lowson, K.A. and Hage, W.J. (1994): Clonal analysis of the origin of primordial germ cells in the mouse. Ciba found. Symp., 182, 68-84.

4) Tam, P.P. and Zhou, S.X. (1996): The allocation of epiblast cells to ectodermal and germ-line lineages is influenced by 
the position of the cells in the gastrulating mouse embryo. Dev. Biol., 178, 124-132.

5) Ginsburg, M., Snow, M.H. and McLaren, A. (1990): Primordial germ cells in the mouse embryo during gastrulation. Development, 110, 521-528.

6 Yeom, Y.I., Fuhrmann, G., Ovitt, C.E. , Brehm, A., Ohbo, K., Gross, M., Hubner, K. and Scholer, H.R. (1996): Germline regulatory element of Oct-4 specific for the totipotent cycle of embryonal cells. Development, 122, 881-894.

7) Lasko, P.F. and Ashburner, M. (1988): The product of the Drosophila gene vasa is very similar to eukaryotic factor4A, Nature, 335, 611-617.

8) Toyooka, Y., Tsunekawa, N., Takahashi, Y., Matsui, Y., Satoh, M. and Noce, T. (2000): Expression and intracellular localization of mouse Vasa-homologue protein during germ cell development. Mech. Dev., 93, 139-149.

9) Castrillon, D.H., Quade, B.J., Wang, T.Y., Quigley, C. and Crum, C.P. (2000): The human VASA gene is specifically expressed in the germ cell lineage. Proc. Natl. Acad. Sci. USA, 97, 9585-9590.
10) Lowson, K.A., Dunn, N.R., Roelen, B.A. Zeinstra, L.M., Davis, A.M., Wright, C.V., Korving, J.P. and Hogan, B.L. (1999): Bmp4 is required for the generation of primordial germ cells in the mouse embryo. Genes Dev., 13, 424-436.

11) Brinster, R.L. and Zimmerman, J.W. (1994): Spermatogenesis following male germ cell transplantation. Proc. Natl. Acad. Sci. USA, 91, 11298-11302.

12) Brinster, R.L. (2002): Germline stem cell transplantation and transgenesis. Science, 296, 2174-2176.

13) Feng, L-X., Chen Y., Dettin, L., Reijo Pera, R.A., Herr, J.C, Goldberg, E. and Dym, M. (2002): Generation and in vitro differentiation of a spermatogonial cell line. Science, 297, 392-395.

14) Rassoulzadegan, M., Paquis-Flucklinger, V., Bertino, B., Sage, J., Jasin, M., Miyagawa, K., Van heyningen, V., Besmer, P. and Cuzin, F. (1993): Transmeiotic differentiation of male germ cells in culture. Cell, 75, 9971006.

15) Honaramooz, A., Snedaker, A., Boiani, M., Scholer, H., Dobinski, I. and Schlatt, S. (2002): Sperm from neonatal mammalian testes grafted in mice. Nature, 418, 778-781. 